\title{
Biocronología de la Terraza Compleja de Butarque del río Manzanares en el Estanque de Tormentas al sur de Madrid (España)
}

\author{
Biochronology of the Complex Terrace of Butarque of the \\ Manzanares river at the Stormwater Tank in southern \\ Madrid (Spain)
}

\author{
C. Laplana ${ }^{1}$, E. Herráez ${ }^{2}$, J. Yravedra Saínz de los Terreros ${ }^{3}$, S. Bárez ${ }^{4}$, S. Rubio-Jara ${ }^{5}$, \\ J. Panera ${ }^{5}$, I. Rus ${ }^{6}$, A. Pérez-González \\ 1 Museo Arqueológico Regional de la Comunidad de Madrid, Pza. de las Bernardas s/n, 28801 Alcalá de Henares \\ (Madrid, España). Email: cesar.laplana.conesa@madrid.org \\ 2 Geolineal SL, Calle la Mira, 12, 28240 Hoyo de Manzanares, Madrid (España) \\ 3 Departamento de Prehistoria, Universidad Complutense de Madrid, C/ Prof. Aranguren s/n 28040 Madrid (España) \\ 4 Departamento de Geodinámica, Universidad Complutense de Madrid, C/ José Antonio Novais 2, 28040 Madrid (España) \\ 5 IDEA, Instituto de Evolución en África, Museo de los Orígenes, Pza. de San Andrés 2, 28005 Madrid (España) \\ 6 Dirección General de Patrimonio Histórico, Comunidad de Madrid, C/ Arenal 18, 28013 Madrid (España) \\ 7 CENIEH, Centro Nacional de Investigación sobre la Evolución Humana, Paseo de la Sierra de Atapuerca 3, 09002 Burgos \\ (España)
}

\section{RESUMEN}

Con motivo de la construcción en 2006 del Estanque de Tormentas en Villaverde (Madrid) se localizaron dos nuevos yacimientos de vertebrados del Pleistoceno, denominados $\mathrm{H}-02$ y $\mathrm{H}-03$, en depósitos de la Terraza Compleja de Butarque (TCB). Las dataciones realizadas por Termoluminiscencia (TL) situaron al primero de los yacimientos $\mathrm{H}-02$ en el Pleistoceno Superior, al final del MIS 5, mientras que para el yacimiento $\mathrm{H}-03$ se obtuvo una edad más antigua, ya dentro del Pleistoceno Medio (Domínguez-Alonso et al., 2009). En este trabajo se evalúa la concordancia de estas dataciones con la estimación de la edad obtenida a partir de la interpretación biocronológica de las asociaciones de mamíferos fósiles recuperadas en cada uno de los yacimientos. Mientras que para el yacimiento $\mathrm{H}-03$ existe congruencia entre la datación por TL y la estimación de su edad a partir de criterios biocronológicos, que combinadas sitúan al yacimiento en la segunda mitad del Pleistoceno Medio, no ocurre lo mismo en el caso de H-02. En este yacimiento, la presencia de Microtus brecciensis señala una edad más antigua que la propuesta por las dataciones de TL, dentro del Pleistoceno Medio. La comparación de algunas características biométricas entre las poblaciones de esta última especie de $\mathrm{H}-02$ y de Áridos 1 permite además precisar que la edad del primero es más reciente que el segundo. Teniendo en cuenta que las dataciones disponibles para Áridos 1 (Panera et al., 2011) sitúan este yacimiento a finales del MIS 11, el yacimiento H-02 tuvo que formarse en el último cuarto del Pleistoceno Medio. Por consiguiente, las dataciones obtenidas por TL para el yacimiento H-02 parecen corresponder con edades mínimas de los sedimentos y no con su edad real.

Palabras clave: Terraza Compleja de Butarque; Biocronología; Pleistoceno Medio; Microtus (Iberomys) brecciensis; Región central de la península ibérica.

Recibido el 18 de junio de 2014 / Aceptado el 2 de febrero de 2015 / Publicado online el 13 de mayo de 2015

Citation / Cómo citar este artículo: C. Laplana et al. (2015). Biocronología de la Terraza Compleja de Butarque del río Manzanares en el Estanque de Tormentas al sur de Madrid (España). Estudios Geológicos 71(1): e028. http://dx.doi.org/10.3989/egeol.41808.338.

Copyright: ( 2015 CSIC. This is an open-access article distributed under the terms of the Creative Commons Attribution-Non Commercial (by-nc) Spain 3.0 License. 
C. Laplana et al.

\begin{abstract}
Due to the construction of the Stormwater Tank in Villaverde (Madrid), two new Pleistocene vertebrate bearing sites were discovered within the sediments of the Complex Terrace of Butarque (TCB, in Spanish), called $\mathrm{H}-02$ and $\mathrm{H}-03$. Termoluminiscence (TL) dates placed the first site at the Late Pleistocene, more precisely at the end of the MIS 5. An older age within the Middle Pleistocene was obtained for H-03 (Domínguez-Alonso et al., 2009). In this work, we evaluate the agreement between the TL dates and the estimated ages resulting from the biochronological interpretation of the fossil mammal assemblages recovered in both sites. Whereas for $\mathrm{H}-03$ there is consistency between the TL dating and the age estimated based on biocronological criteria, this is not the case for $\mathrm{H}-02$. At this site, the presence of Microtus brecciensis points to an older age than the proposal based on $\mathrm{TL}$, within the Middle Pleistocene. On the other hand, comparison of some biometric features between the $\mathrm{H}-02$ and Áridos 1 populations of the last species allows to establish a younger age for the first site with regard to the second one. Taking into account the available dates for Áridos 1, which placed this site at the end of the MIS 11 (Panera et al., 2011), the age of $\mathrm{H}-02$ must therefore be included in the last quarter of the Middle Pleistocene. In summary, TL dates for the $\mathrm{H}-02$ site and, perhaps, some others obtained for the Stormwater Tank in Villaverde, seem to be minimum estimations of the age of the sediments, and do not represent their real age.
\end{abstract}

Keywords: Complex Terrace of Butarque; Biochronology; Middle Pleistocene; Microtus (Iberomys) brecciensis; Central Iberia.

\section{Introducción}

La Terraza Compleja de Butarque (en adelante TCB), define una unidad morfoestratigráfica del valle de río Manzanares, aguas abajo de Madrid, sujeta a procesos de subsidencia sinsedimentaria por disolución de los yesos miocenos infrayacentes que conforman el sustrato (karst subyacente). Se extiende (Fig. 1) desde unos $2 \mathrm{~km}$ aguas arriba de la desembocadura del arroyo de Butarque hasta la confluencia del río Manzanares con el río Jarama en la llanura aluvial de Arganda del Rey (Goy et al., 1989).

Los espesores derivados de los fenómenos de karst subyacente en el tramo final del río Manzanares son de decenas de metros (Uribelarrea, 2008), anómalos si se comparan con las potencias de unos pocos metros de las terrazas escalonadas de aguas arriba, al igual que ocurre en otros valles sujetos a estos procesos en España. En el valle del río Jarama, aguas abajo de Velilla de San Antonio, Pérez-González (1971) describió procesos similares que afectaban a las terrazas del Pleistoceno Medio de $+30 \mathrm{~m}$ y +20 m, quedando agrupadas en la Terraza Compleja de Arganda (Portero \& Pérez-González, 1990; Pérez-González \& Uribelarrea, 2002; y Panera et al., 2011). Asociados a las secuencias sedimentarias de las terrazas complejas de los valles del Manzanares y Jarama existe un gran número de yacimientos arqueo-paleontológicos de edad Pleistoceno Medio o Superior (Santonja et al., 1980; Rus \& Vega, 1984; Rubio Jara et al., 2002; Sesé \& Soto, 2002a y b; Panera et al., 2005; Panera et al., 2011; Yravedra et al., 2012; y Silva et al., 2012).
Con motivo del vaciado realizado para la construcción del Estanque de Tormentas de Butarque (en adelante ETB) en Villaverde (Fig. 1) en 2006 se excavaron nuevos yacimientos pleistocenos en la TCB, denominados H-02 y H-03 (Fig. 2), en los que se ha recuperado una amplia muestra de industria lítica y de restos de vertebrados (Álvarez Catalán et al., 2009; Arcos Fernández et al., 2008, 2011). Dataciones realizadas mediante termoluminiscencia sobre cuarzo por Quaternary TL Surveys en los sedimentos de ambos yacimientos sitúan al primero (H-02) hacia el final del primer tercio del Pleistoceno Superior, mientras que asignan al segundo (H-03) una edad imprecisa dentro del Pleistoceno Medio (Domínguez Alonso et al., 2009) (Figs. 2 y 3).

En este trabajo se presentan las asociaciones de mamíferos de los yacimientos $\mathrm{H}-02$ y $\mathrm{H}-03$, se analiza el significado cronológico de las mismas, y se valora su concordancia o discordancia con respecto a las dataciones numéricas obtenidas en ellos.

\section{Contexto geológico de los nuevos yacimientos de la TCB en el Estanque de Tormentas de Butarque (ETB)}

Los trabajos de construcción del ETB generaron un vaciado de 7 ha de superficie y $30 \mathrm{~m}$ de profundidad máxima (Fig. 3). En este hueco y en su talud oeste, de dirección N-S, DomínguezAlonso et al. (2009; Fig. 2) definieron con criterios arqueológicos, de arriba abajo, tres secuencias estratigráficas que denominaron secuencias 1,2 y 3 , más una cuarta basal formada por un residuo kárstico. 


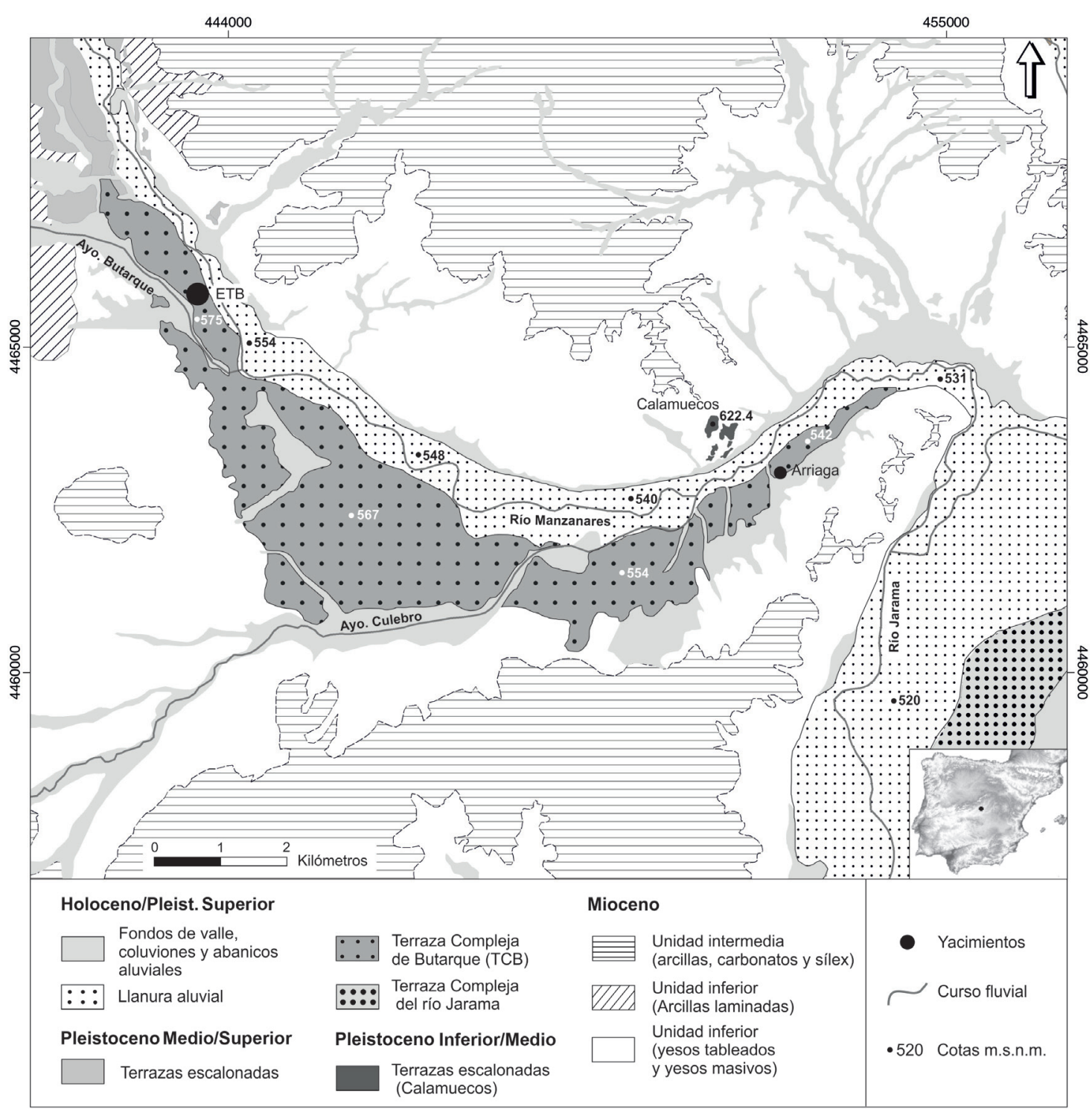

Fig. 1.-La Terraza Compleja de Butarque (TCB) del río Manzanares al sur de Madrid y localización de los yacimientos H-02 y H-03, en el Estanque de Tormentas de Butarque (ETB). En el ángulo inferior derecho, la ubicación de Madrid en la península ibérica.

Igualmente figuraron dos terrazas fluviales del río Manzanares a $+12 \mathrm{~m}$ y $+8-10 \mathrm{~m}$, encajadas entre sí, estando la más alta también solapada con las secuencias anteriores de la ETB, que son por lo tanto más antiguas (Fig. 2).

La secuencia basal 4 está constituida por un residuo de facies limo-arcillosa con intercalaciones de arenas micáceas, de estructura bandeada con concreciones de carbonato y cristalizaciones de yeso secundario, que es el resultado de los procesos de disolución in situ de los yesos infrayacentes miocenos, de edad Pleistoceno según Uribelarrea (2008).
El resto de las secuencias sedimentarias están estructuradas por numerosos ciclos fluviales granodecrecientes de gravas y arenas de facies canalizadas que finalizan con términos de limos y arcillas-arenosas de llanura de inundación.

Durante la construcción del ETB se excavaron dos yacimientos arqueopaleontológicos pleistocenos: H-02 y H-03.

El yacimiento $\mathrm{H}-02$ se localiza cerca de la base de la secuencia 2 (Domínguez-Alonso et al., 2009; Fig. 2). De acuerdo con De los Arcos et al. (2008) y Álvarez-Catalán et al. (2009), los restos 


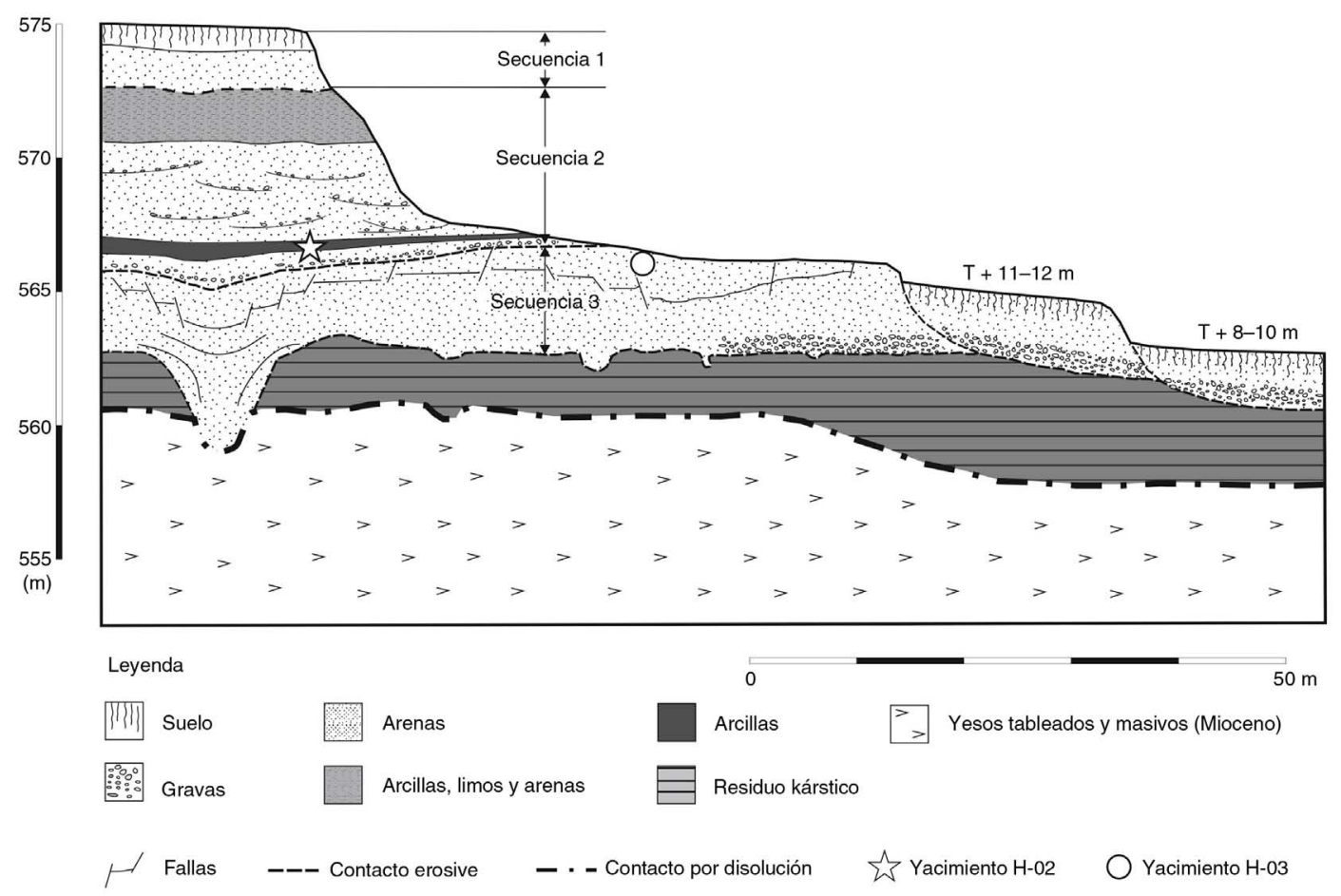

Fig. 2.-Sección morfoestratigráfica (modificada de Domínguez-Alonso et al., 2009, Fig. 2) con las secuencias sedimentarias diferenciadas y posición de los yacimientos $\mathrm{H}-02$ y H-03, y terrazas encajadas ( $\mathrm{T}$ ) del río Manzanares. El yacimiento $\mathrm{H}-03$ en la secuencia 3 ha sido datado por TL en más de $125 \mathrm{ka}$. El yacimiento H-02 a muro de la secuencia 2 arrojó en dos muestras una edad también por TL de 84,6+12,6/-11.2 ka y 74,9+10,2/-9,2 ka. Por último, del techo de la secuencia 2 formada por arcillas, limos y arenas, se obtuvo una edad por TL de $56,8 \pm 4 \mathrm{ka}$.

paleontológicos proceden de un paquete de limos arenosos de $1 \mathrm{~m}$ de potencia y de otro de arcillas de $0,5 \mathrm{~m}$ de espesor situado inmediatamente

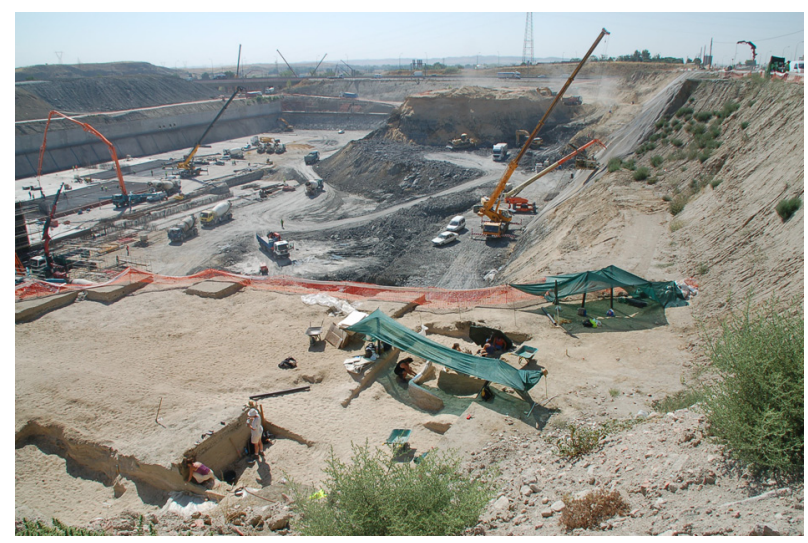

Fig. 3.-Trabajos de vaciado del Estanque de Tormentas de Butarque (ETB) durante los años 2006-2007, y excavaciones arqueológicas en las secuencias 2 y 3 de Domínguez Alonso et al. (2009; Fig. 2). En primer término el yacimiento $\mathrm{H}-02$, situado en la secuencia 2 . por encima del anterior, ambos intercalados entre depósitos de arenas y gravas (Fig. 3). Estos niveles de limos y arcillas de llanura de inundación han sido muestreados en distintos puntos, y como resultado se ha obtenido un conjunto amplio de restos de macro y microvertebrados, así como de industria lítica. Dos dataciones por TL del yacimiento H-02 arrojaron valores de 84,6+12,6/-11,2 ka y 74,9+10,2/-9,2 ka (Domínguez Alonso et al., 2009; Fig. 3).

El yacimiento $\mathrm{H}-03$ se encuentra a techo de la secuencia 3 en este sector de la TCB. Los restos paleontológicos proceden de unos niveles de limos verdosos con pequeños lentejones de arcillas de tono verde oscuro que representan facies canalizadas someras de baja energía. Estos niveles han sido igualmente muestreados en distintos puntos, lo que ha permitido obtener un reducido conjunto de restos de microvertebrados y otro, más amplio, de grandes mamíferos. Una muestra arenosa de la secuencia 3 , donde se sitúa este yacimiento, ha 
proporcionado una edad por TL de $>125 \mathrm{ka}$ BP (Domínguez Alonso et al., 2009).

Por otra parte, hay que señalar que DomínguezAlonso et al. (2009; Figs. 2 y 3 ) asignan una cronología por TL de $56,8 \pm 4$ ka para el techo de la secuencia 2, sobre la que se dispone la secuencia 1 de arenas y arcillas fluviales de origen no determinado por los citados autores, con ocupaciones en su parte superior neolíticas, de la edad del bronce, hierro y alto medieval. Su edad comprendería probablemente desde el Pleistoceno Superior final hasta el Holoceno.

\section{Mamíferos de los yacimientos H-02 y H-03}

Los listados de las especies de mamíferos identificadas en los nuevos yacimientos del ETB se encuentran en la Tabla 1. Como se aprecia en dicha tabla, ambos yacimientos tienen en común un amplio conjunto de taxones. La descripción detallada de los materiales correspondientes a estas especies será motivo de una serie de trabajos posteriores, cuando finalice el proceso de preparación para su estudio, sobre todo de los restos de grandes mamíferos. Todos los materiales se encuentran depositados en el Museo Arqueológico Regional de la Comunidad de Madrid. A continuación se señalan brevemente algunos aspectos relativos a las diferencias entre las asociaciones de los dos yacimientos y, cuando es necesario, se realizan algunas puntualizaciones sobre la identificación de las especies.

Entre los mamíferos de talla grande, son elementos comunes a los dos yacimientos las especies Stephanorhinus sp., Equus ferus, Equus hydruntinus, Bos primigenius y Cervus elaphus. H-02 cuenta además con restos de Canis lupus, Sus scrofa y Bison priscus. En el caso de los grandes bóvidos, la distinción entre Bison y Bos resulta frecuentemente problemática si no se dispone de los elementos diagnósticos. En H-02 estos bóvidos están representados por mandíbulas con la dentición completa y por metatarsos, lo que ha permitido confirmar la presencia de ambos géneros. A estas especies hay que añadir varios fragmentos de cráneo, un premaxilar, un metapodio y una defensa de proboscídeo. En algunos trabajos previos (Álvarez-Lao \& García, 2010, 2012; Álvarez-Lao, 2014) se señala a partir de este material la presencia en $\mathrm{H}-02$ de Mammuthus

\begin{tabular}{|c|c|c|}
\hline & $\mathrm{H}-02$ & $\mathrm{H}-03$ \\
\hline \multicolumn{3}{|l|}{ Orden ERINACEOMORPHA } \\
\hline Erinaceus sp. & $x$ & - \\
\hline \multicolumn{3}{|l|}{ Orden SORICOMORPHA } \\
\hline Crocidura cf. C. russula & cf. & - \\
\hline \multicolumn{3}{|l|}{ Orden RODENTIA } \\
\hline Eliomys quercinus & $\mathrm{x}$ & - \\
\hline Allocricetus bursae & $\mathrm{x}$ & - \\
\hline Arvicola cf. A. sapidus & cf. & sp. \\
\hline Microtus arvalis & $\mathrm{x}$ & - \\
\hline Microtus brecciensis & $x$ & - \\
\hline Microtus sp. & - & $x$ \\
\hline Apodemus sp. gr. A. sylvaticus-A. flavicollis & $\mathrm{x}$ & $x$ \\
\hline \multicolumn{3}{|l|}{ Orden LAGOMORPHA } \\
\hline Oryctolagus cuniculus & $x$ & $x$ \\
\hline Lepus sp. & $\mathrm{x}$ & - \\
\hline \multicolumn{3}{|l|}{ Orden CARNIVORA } \\
\hline Canis lupus & $\mathrm{X}$ & - \\
\hline \multicolumn{3}{|l|}{ Orden PROBOSCIDEA } \\
\hline Palaeoloxodon antiquus & $\mathrm{X}$ & - \\
\hline \multicolumn{3}{|l|}{ Orden PERISSODACTYLA } \\
\hline Stephanorhinus sp. & $\mathrm{x}$ & $X$ \\
\hline Equus ferus & $\mathrm{X}$ & $x$ \\
\hline Equus hydruntinus & $\mathrm{X}$ & $x$ \\
\hline \multicolumn{3}{|l|}{ Orden ARTIODACTYLA } \\
\hline Sus scrofa & $\mathrm{X}$ & - \\
\hline Cervus elaphus & $\mathrm{X}$ & $\mathrm{X}$ \\
\hline Bos primigenius & X & $\mathrm{X}$ \\
\hline Bison priscus & $\mathrm{X}$ & - \\
\hline
\end{tabular}

primigenius. Sin embargo, el análisis de las líneas y ángulos de Schreger del esmalte de la defensa conservada (Fig. 4), según el método propuesto por Espinoza \& Mann (1993) realizado por Ros Montoya (2010), lo identifica como Palaeoloxodon antiquus. Por ello, en este trabajo se considera que la especie de proboscídeo presente en $\mathrm{H}-02$ es $P$. antiquus y no M. primigenius.

A pesar de que la preservación de los huesos no es buena, en H-03 se han observado algunas marcas de corte sobre los huesos de ciervo, caballo y uro, que indicarían algunos episodios de intervención 


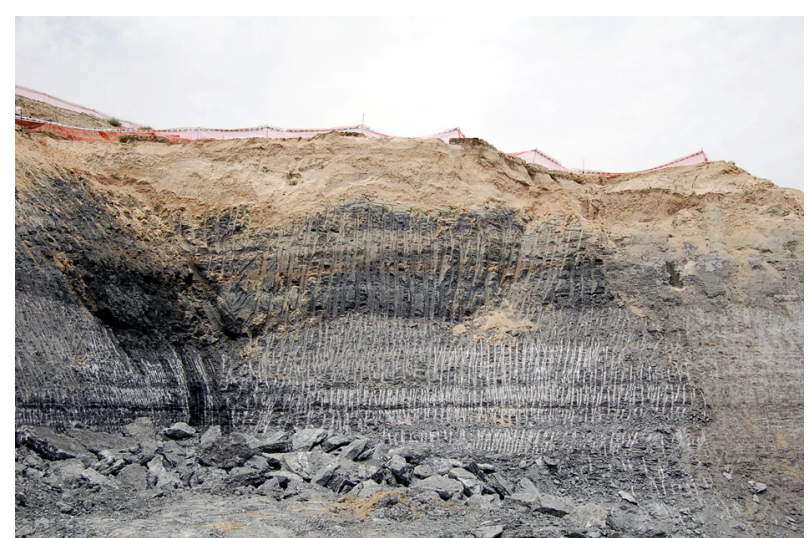

Fig. 4.-Estratigrafía del frente este del vaciado del Estanque de Tormentas de Butarque. En la parte inferior los yesos tableados y masivos miocenos y a su techo el residuo kárstico in situ por disolución de los yesos infrayacentes (edad Pleistoceno Medio, Uribelarrea, 2008). En posición superior el muro arenoso de la secuencia 3 depositada por el río Manzanares, contiene a su techo el yacimiento $\mathrm{H}-03$ de Domínguez-Alonso et al. (2009, Fig. 2).

humana sobre la fauna (Yravedra, 2006). No se aprecia sobre los restos de fauna de tamaño grande o pequeño ninguna evidencia (p. e., diferencias en la coloración entre elementos de distintas especies, pulimentos o abrasiones que indiquen transporte de los materiales, ...) que sustente la existencia de fenómenos de mezcla de materiales de distintas cronologias.

Todas las especies de grandes mamíferos identificadas en los yacimientos del ETB son frecuentes en las terrazas del Manzanares y del Jarama (Sesé \& Soto, 2002a y b).

Respecto al registro de micromamíferos, este es mucho más abundante en $\mathrm{H}-02$ que en $\mathrm{H}-03$, debido en parte al diferente volumen de sedimento procesado. Los restos de micromamíferos proceden de muestras tomadas en distintos puntos de cada uno de los yacimientos, lavadas con una pila de tamices superpuestos de luces de malla decrecientes, la menor de las cuales es de $0,5 \mathrm{~mm}$. La asociación de H-02 se obtuvo del lavado de 8250 kgr de sedimento. Inicialmente, Herráez Igualador \& Álvarez Catalán (2007) identificaron en este yacimiento las siguientes especies de micromamíferos: Crocidura cf. C. russula, Allocricetus bursae, Arvicola aff. A. sapidus, Microtus brecciensis, Apodemus sylvaticus, Eliomys quercinus, Oryctolagus cf. O. cuniculus. Más tarde, Arcos Fernández et al. (2008) añadieron
Erinaceus sp. al listado de micromamíferos del yacimiento. La revisión de la colección de micromamíferos de $\mathrm{H}-02$ realizada en este trabajo ha supuesto la adición de algunos taxones a los listados propuestos anteriormente (Microtus arvalis, Lepus sp.) así como la modificación de algunas de las identificaciones previas (ver Tabla 2). En H-03, se procesaron $1080 \mathrm{kgr}$ de sedimento, y las especies identificadas son Arvicola sp., Microtus sp., Apodemus sp. gr. A. sylvaticus-A. flavicollis y Oryctolagus cuniculus (Herráez Igualador \& Álvarez Catalán, 2007).

\section{Significado biocronológico de las asociaciones de mamíferos de los yacimientos $\mathrm{H}-02$ y H-03 del ETB}

La asociación del yacimiento $\mathrm{H}-03$ está constituida por un reducido número de taxones de grandes y pequeños mamíferos, lo que limita las precisiones cronológicas que pueden ser obtenidas a partir de la misma. Todas ellas se encuentran presentes tanto en los yacimientos de la segunda mitad del Pleistoceno Medio como del Pleistoceno Superior de las terrazas del Manzanares y del Jarama (Sesé \& Soto, 2002a y b). Por lo tanto, la edad que se puede proponer para $\mathrm{H}-03$ a partir de su listado faunístico estaría entre el inicio de la segunda mitad del Pleistoceno Medio y el final del Pleistoceno Superior.

El yacimiento $\mathrm{H}-02$ cuenta con una asociación más rica en número de especies, algunas de las cuales son muy significativas desde el punto de vista biocronológico y permiten acotar más precisamente su edad.

El elemento que proporciona más información sobre la cronología de este yacimiento es Microtus brecciensis, ya que la evolución a lo largo del Pleistoceno del subgénero Iberomys al que pertenece ha sido estudiada por distintos autores (Ayarzagüeña \& López-Martínez, 1976; Cabrera-Millet et al., 1982; Cuenca-Bescós \& Laplana, 1995; Paunesco \& Brunet-Lecomte, 2005, entre otros). Esta especie es además la más abundante en número de restos en H-02, mientras que la escasez de restos de las demás especies impone limitaciones a la interpretación biocronológica de sus características morfológicas y biométricas.

Durante el Pleistoceno Medio, el subgénero Iberomys se encuentra representado por la especie $M$. brecciensis, antecesora de la especie actual 
Tabla 2.-Longitud del primer molar inferior (con indicación de los valores mínimo, medio y máximo, la desviación estándar de la muestra y el número de elementos) de las poblaciones ibéricas del subgénero Iberomys durante el Pleistoceno y Holoceno (solo se han incluido aquellas con más de 10 ejemplares) y de dos poblaciones actuales de M. cabrerae. Datos procedentes de: 1) Engels (1972); 2) Ayarzagüeña \& López Martínez (1976); 3) López Martínez (1980); 4) Ruiz Bustos et al. (1982); 5) Gil (1985); 6) Gil (1988); 7) Povoas et al. (1992); 8) Gil (1997); 9) Laplana \& Cuenca-Bescós (1998); 10) Jeannet (2000); 11) Ruiz Bustos (2000); 12) Barroso Ruiz et al. (2006); 13) Guillem Calatayud (2009); 14) Tormo Cuñat (2010); 15) López-García (2011); 16) Sesé et al. (2011a); 17) Sesé et al. (2011b); 18) Hanquet (2011); 19) Price (2012); 20) Laplana \& Sevilla (2013); 21) Cuenca-Bescós et al. (2014); 22) este trabajo. Dentro de cada intervalo temporal, los yacimientos han sido ordenados cronológicamente cuando ha sido posible. Se aprecia con claridad el salto biométrico entre las poblaciones del Pleistoceno Medio asignadas a $\mathbf{M}$. brecciensis, con longitudes medias inferiores a 2,95 mm, y las del Pleistoceno Superior y Holoceno, pertenecientes a M. cabrerae, con longitudes medias superiores a 3,05 mm

\begin{tabular}{|c|c|c|c|c|c|c|c|}
\hline \multirow[b]{2}{*}{ Intervalo temporal } & \multirow[b]{2}{*}{ Localidad/Yacimiento } & \multicolumn{5}{|c|}{ Longitud del primer molar inferior } & \multirow[b]{2}{*}{ Especies } \\
\hline & & $\min$ & $\mathrm{m}$. & $\max$ & d.e. & $\mathrm{n}$ & \\
\hline \multirow[t]{2}{*}{ Actualidad } & Porto Covo (Portugal) $^{1}$ & 2,81 & 3,28 & 3,75 & 0,21 & 27 & M. cabrerae \\
\hline & Cuenca (España) $)^{2}$ & 2,96 & 3,32 & 3,48 & 0,16 & 25 & \\
\hline \multirow[t]{3}{*}{ Holoceno } & Valdetorres de Jarama ${ }^{20}$ & 2,80 & 3,13 & 3,60 & 0,20 & 13 & \\
\hline & Cova del Toll ${ }^{10}$ & 2,96 & 3,24 & 3,67 & 0,17 & 22 & \\
\hline & Cova de les Cendres, Holoc. ${ }^{13}$ & 2,95 & 3,28 & 3,70 & 0,05 & 13 & \\
\hline \multirow[t]{9}{*}{ Pleistoceno Superior } & Gruta do Caldeirao niv. K-E ${ }^{7}$ & 2,65 & 3,21 & 3,55 & 0,20 & 20 & \\
\hline & Cova de les Cendres, Gravet. ${ }^{14}$ & 3,11 & 3,37 & 3,84 & - & 16 & \\
\hline & Cueva de las Graderas ${ }^{6}$ & 2,86 & 3,25 & 3,68 & - & 58 & \\
\hline & Figueira Brava $^{10}$ & 2,70 & 3,09 & 3,46 & 0,21 & 15 & \\
\hline & Abric Romaní ${ }^{15}$ & 3,00 & 3,35 & 3,72 & 0,19 & 25 & \\
\hline & Boquete de Zafarraya $^{12}$ & 2,81 & 3,28 & 3,64 & - & 93 & \\
\hline & Gorham's Cave SSLm ${ }^{19}$ & 2,86 & 3,26 & 3,56 & 0,18 & 28 & \\
\hline & Cueva de la Carihuela niv. VIII & 2,73 & 3,10 & 3,51 & - & 11 & \\
\hline & Preresa $^{17}$ & 2,81 & 3,10 & 3,51 & - & 50 & \\
\hline \multirow[t]{7}{*}{ Pleistoceno Medio } & Sala de los Huesos ${ }^{18}$ & 2,56 & 2,93 & 3,20 & 0,22 & 16 & M. brecciensis \\
\hline & H-02 Butarque 22 & 2,57 & 2,86 & 3,10 & 0,14 & 11 & \\
\hline & Valdocarros $^{16}$ & 2,67 & 2,88 & 3,17 & - & 17 & \\
\hline & Cueva del Agua ${ }^{4}$ & 2,60 & 2,92 & 3,36 & - & 90 & \\
\hline & Áridos-1 ${ }^{3}$ & 2,32 & 2,79 & 3,18 & 0,24 & 44 & \\
\hline & TD8 $^{8}$ & 2,64 & 2,82 & 3,08 & - & 11 & \\
\hline & Cúllar de Baza-I ${ }^{4}$ & 2,55 & 2,94 & 3,27 & - & 37 & \\
\hline \multirow[t]{2}{*}{ Pleistoceno Inferior } & TD6 $^{9}$ & 2,35 & 2,69 & 3,04 & 0,13 & 171 & \\
\hline & $\mathrm{TD}^{21}$ & 2,24 & 2,71 & 3,07 & 0,15 & 85 & \\
\hline
\end{tabular}

M. cabrerae. De acuerdo con los autores citados más arriba, las diferencias entre ambas especies consisten en el menor tamaño de la dentición de la especie más antigua, así como una serie de aspectos morfológicos entre los que destacan la mayor asimetría transversal, menor anchura relativa y menor proporción de ejemplares con BRA4 de los m1, y la mayor confluencia de los triángulos en el m3 en $M$. brecciensis comparado con $M$. cabrerae. En relación a la cronología de la transición entre ambas especies, Laplana \& Sevilla
(2013) realizan un análisis de los datos biométricos publicados y concluyen que en la península ibérica todas las poblaciones del Pleistoceno Medio tienen una talla pequeña propia de $M$. brecciensis (longitud media del $\mathrm{m} 1<2,95 \mathrm{~mm}$ ), mientras que todas las del Pleistoceno Superior y Holoceno tienen una talla mayor (longitud media del $\mathrm{m} 1>3,05 \mathrm{~mm}$ ), similar a la de M. cabrerae actual (Tabla 2). En ocasiones, algunas de las poblaciones del Pleistoceno Superior y del Holoceno han sido identificadas como M. brecciensis, 
incluso recientemente (Cova del Dalt, Olària et al., 2005 y Gusi et al., 2013; Cueva de Aguilón P-7, Cuenca-Bescós et al., 2010a). Sin embargo, a partir del criterio biométrico señalado más arriba así como por sus características morfológicas, en nuestra opinión se deben incluir dentro de la variabilidad de $M$. cabrerae.

En la península ibérica, el registro más moderno de $M$. brecciensis es, probablemente, el de la Sala de los Huesos en la Cueva de Maltravieso, datado dentro del MIS 6 o, con menos probabilidad, al comienzo del MIS 5 (Hanquet, 2011). Por otro lado, los registros más antiguos de $M$. cabrerae son los yacimientos de la Cueva del Camino (= Pinilla del Valle; Toni y Molero, 1990; Laplana et al., 2013), PRERESA (Sesé et al., 2011b) y los del nivel VII de la Cueva de la Carihuela (Ruiz Bustos, 2000), de finales del MIS 5. A estos yacimientos habría que añadir también Arriaga I. Aunque López Martínez (en Gamazo, 1982) identificó en este yacimiento la especie $M$. brecciensis, la elevada longitud de los primeros molares inferiores $(\mathrm{m} 1)$ atribuidos a esta especie (longitud media del $\mathrm{m} 1=3,22 \mathrm{~mm}$ de acuerdo con Sesé \& López Martínez, 2013), fuera del rango de variación de las poblaciones de esta especie de los yacimientos de las terrazas del Jarama y Manzanares y similar a los valores medios en las poblaciones de M. cabrerae actuales y del Pleistoceno Superior (ver Laplana \& Sevilla, 2013) es, en nuestra opinión, indicativa de su pertenencia a $M$. cabrerae en lugar de $M$. brecciensis. Recientemente, este yacimiento ha sido datado por TL en la parte final del Pleistoceno Medio (133-134 ka, son edades mínimas) por Silva et al. (2013). Conviene señalar que, en ausencia de esta datación, la presencia de M. cabrerae en Arriaga I situaría este yacimiento con más probabilidad en el Pleistoceno Superior, ante la ausencia de registro ibérico de esta especie en el Pleistoceno Medio. Sin embargo, la existencia de un vacío de datos biométricos del linaje Iberomys al final del Pleistoceno Medio y al principio del Superior no permite descartar una edad terminal dentro del Pleistoceno Medio para este yacimiento. Si se acepta la edad Pleistoceno Medio para el yacimiento de Arriaga I, este yacimiento contendría el registro más antiguo de $M$. cabrerae en la península ibérica. En resumen, el salto biométrico entre las poblaciones de pequeña talla correspondientes a $M$. brecciensis y el de aquellas otras de talla mayor asignadas a $M$. cabrerae en la península ibérica se produce en torno al tránsito Pleistoceno Medio-Superior.

La población del yacimiento $\mathrm{H}-02$ del Estanque de Tormentas de Butarque, aunque solo cuenta con 11 $\mathrm{m} 1$ completos, se caracteriza por el pequeño tamaño de los mismos (longitud media del $\mathrm{m} 1=2,86 \mathrm{~mm}$; Tabla 2) y por sus terceros molares inferiores (m3) con los triángulos confluentes (Fig. 6), caracteres propios de $M$. brecciensis. Comparada con la población de Áridos 1 (Tabla 3), los m1 de H-02 presentan una anchura relativamente mayor y una asimetría menor, lo que confiere a esta última un carácter más evolucionado. Un conjunto de dataciones por racemización de aminoácidos (AAR) procedentes de la secuencia fluvial de Arganda I en la que se encuentra el yacimiento de Áridos 1 han dado fechas entre aprox. 300-400 ka, con solapamiento de dataciones en el intervalo en torno al final del MIS 11 (entre 335 y 370 ka; Panera et al., 2011). Tomando estas dataciones por AAR como referencia, y teniendo en cuenta el carácter más evolucionado de la población de $M$. brecciensis de $\mathrm{H}-02$ respecto de la de Áridos 1, la edad de H-02 se situaría en el último cuarto del Pleistoceno Medio (130-300 ka).

Esta interpretación cronológica se encuentra apoyada también por la presencia en este yacimiento de $M$. arvalis. Esta especie está ausente en los yacimientos de las terrazas del Jarama y Manzanares que

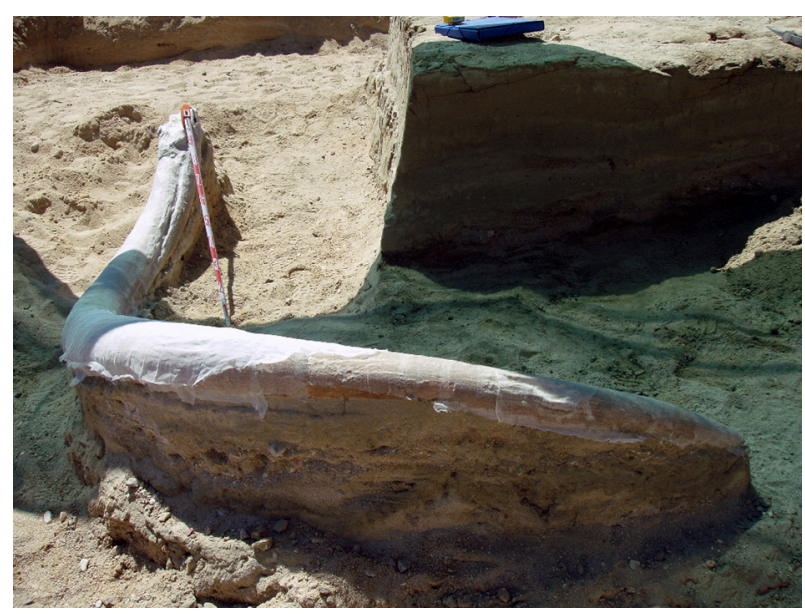

Fig. 5.-Defensa de Palaeoloxodon antiquus, de $3,80 \mathrm{~m}$ de longitud. Yacimiento $\mathrm{H}-02$ en la base de la secuenca 2 (Domínguez-Alonso et al., 2009, Fig. 2) del Estanque de Tormentas de Butarque (ETB). 

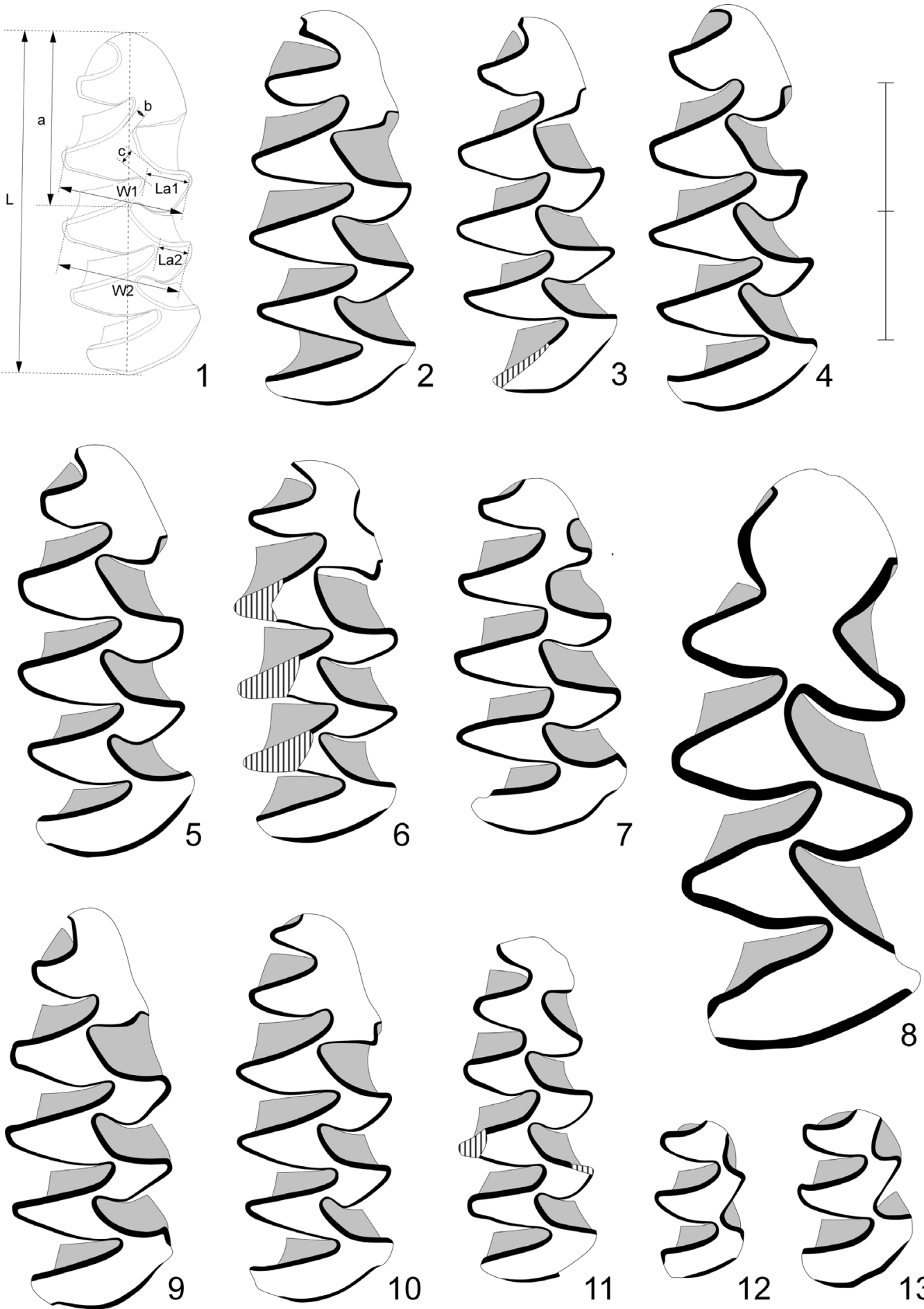

Fig. 6.-1: Esquema de las medidas sobre los primeros molares inferiores de Microtus brecciensis. Los índices de la Tabla 3 se han calculado a partir de las medidas que aparecen en el esquema de la siguiente forma: $A / L=a / L * 100 ; B / W=b / W 1^{*} 100 ; C / W=c / W 1^{*} 100$; AS1 = La1/W1*100; AS2 = La2/W2*100; L/W = L/W1. Las medidas e índices L, W1, a, b, c, A/L, B/W, C/W son las propuestas por van der Meulen (1973), la medida La1 es similar, aunque no idéntica, a la medida La de Cuenca-Bescós et al. (1995). 2 a 13: Arvicolinos del yacimiento H-02 del Estanque de Tormentas de Butarque (Villaverde, Madrid). 2-7 y 9, 10: m1 de Microtus brecciensis; 8) m1 de Arvicola cf. A. sapidus; 12, 13) m3 de Microtus brecciensis; 11) m1 de Microtus arvalis. Todos los molares están dibujados como si fueran derechos, aunque no lo sean. Escala gráfica: $2 \mathrm{~mm}$. 
Tabla 3.-Comparación biométrica de las poblaciones de Microtus brecciensis de Áridos 1 y de H-02. Las medidas han sido tomadas de acuerdo con el esquema de la Fig. 6.1. Las medidas $L$ y W se expresan en milímetros, los índices se expresan como porcentajes, salvo el índice L/W, que es adimensional. La última columna expresa el valor del estadístico p resultado de aplicar el test de Student, o de Mann-Whitney o de permutaciones en la comparación de las medias o medianas, en función de las características de las muestras (normalidad y homocedasticidad). Los cálculos han sido realizados con el programa Past v. 2.17 (Hammer et al., 2001). Cuando este valor es inferior a 0,05, las muestras difieren entre sí con una probabilidad del $95 \%$. En sombreado se resaltan aquellas comparaciones con resultado de diferencias estadísticamente significativas

\begin{tabular}{|c|c|c|c|c|c|c|c|c|c|c|c|}
\hline & \multicolumn{5}{|c|}{$\mathrm{H}-02$} & \multicolumn{5}{|c|}{ Áridos 1} & \multirow[b]{2}{*}{ p-valo } \\
\hline & $\mathrm{n}$ & $\min$ & $\mathrm{m}$ & $\max$ & DS & $\mathrm{n}$ & $\min$ & $\mathrm{m}$ & $\max$ & DS & \\
\hline $\mathrm{L}$ & 11 & 2,57 & 2,86 & 3,10 & 0,16 & 19 & 2,39 & 2,90 & 3,17 & 0,21 & 0,5116 \\
\hline W & 11 & 1,03 & 1,14 & 1,25 & 0,06 & 19 & 0,92 & 1,04 & 1,16 & 0,06 & 0,0001 \\
\hline $\mathrm{A} / \mathrm{L}$ & 11 & 45,42 & 50,71 & 54,53 & 2,56 & 19 & 40,53 & 51,90 & 55,34 & 3,33 & 0,3354 \\
\hline $\mathrm{B} / \mathrm{W}$ & 11 & 1,86 & 5,43 & 20,87 & 6,32 & 19 & 1,49 & 2,27 & 3,31 & 0,69 & 0,0562 \\
\hline $\mathrm{C} / \mathrm{W}$ & 11 & 3,00 & 3,88 & 5,24 & 0,77 & 19 & 1,72 & 4,26 & 12,0 & 2,75 & 0,4483 \\
\hline $\mathrm{AS}_{1}$ & 11 & 28,95 & 33,78 & 38,36 & 2,74 & 19 & 22,61 & 29,34 & 31,78 & 2,43 & 0,0002 \\
\hline $\mathrm{AS}_{2}$ & 7 & 27,74 & 32,37 & 36,62 & 2,98 & 17 & 26,09 & 30,06 & 32,84 & 1,90 & 0,0325 \\
\hline L/W & 8 & 2,30 & 2,52 & 2,70 & 0,14 & 19 & 2,46 & 2,79 & 3,03 & 0,17 & 0,0004 \\
\hline
\end{tabular}

incluyen también en sus asociaciones $M$. brecciensis, como Áridos 1 o Valdocarros. En Arriaga I, Sesé \& López Martínez (2013) describen una asociación con $M$. cabrerae (originalmente identificado como M. brecciensis; ver discusión más arriba) y $M$. arvalis. Por consiguiente, $M$. arvalis es una especie que se incorpora muy tardíamente (al final del Pleistoceno Medio) a las asociaciones de micromamíferos de los yacimientos de las terrazas del Jarama y Manzanares, siendo la de H-02 la cita más antigua de la especie en este contexto geográfico. La presencia de $M$. arvalis en $\mathrm{H}-02$ es evidencia por tanto de que la edad de este yacimiento es más reciente que Áridos 1 y Valdocarros, en donde esta especie está ausente. Por otro lado, la presencia de $M$. brecciensis en $\mathrm{H}-02$ sugiere que podría ser algo más antiguo que Arriaga I, que incluye un representante del subgénero Iberomys con $\mathrm{m} 1$ de talla netamente mayor, fuera del rango de variación de los $\mathrm{m} 1$ de M. brecciensis de H-02 y que, como hemos indicado más arriba, en nuestra opinión debe ser identificado como M. cabrerae.

Desde el punto de vista climático, la asociación de mamíferos de $\mathrm{H}-02$ sugiere unas condiciones climáticas menos cálidas que las que existieron durante la formación de otros yacimientos de las terrazas del Jarama y Manzanares. Esta interpretación se basa en la presencia en este yacimiento de $M$. arvalis entre los micromamíferos y de B. priscus entre los grandes mamíferos.
M. arvalis es una especie característica de los sistemas montañosos de la mitad norte y centro de la península ibérica (Rey, 1973). En la actualidad ocupa también toda la meseta norte (González Esteban \& Villate, 2007), debido a que los cambios en el uso del territorio llevados a cabo en los últimos 40 años por el ser humano han propiciado que haya colonizado las tierras bajas a lo largo de todo este ámbito geográfico (Luque-Larena et al., 2013). Durante el Pleistoceno Medio, su presencia estuvo limitada al tercio norte peninsular, y solo tal vez al final de este período comenzó a registrarse en regiones más al sur (Arriaga; Sesé \& López-Martínez, 2013 - ver discusión sobre la edad de este yacimiento más arriba). En la segunda mitad del Pleistoceno Superior, período en el que el clima fue predominantemente más frío que el actual, alcanzó el sur peninsular, habiendo sido citada en un amplio conjunto de yacimientos andaluces (Cueva de la Carihuela - Ruiz Bustos \& García Sánchez, 1977; Cueva Horá - García, 1979; Cueva de Zafarraya - Medina-Lara et al., 1986; Cueva de los Ojos - Fuentes Jiménez, 1989; Cueva de las Ventanas - Ruiz Bustos \& Riquelme Cantal, 1999; Cueva del Cucú - González-Ramón et al., 2012). Con el mejoramiento climático holoceno el área de distribución de la especie sufrió un retroceso hacia el norte, quedando confinada a los sistemas montañosos septentrionales, hasta su reciente expansión debida a factores antrópicos. M. arvalis es por consiguiente una especie sensible a los cambios 
climáticos, ampliando en la península su distribución hacia el sur en los momentos más fríos y restringiéndola hacia el norte en los más cálidos. Su presencia en $\mathrm{H}-02$, yacimiento de fondo de valle del centro peninsular del último cuarto del Pleistoceno Medio, marca el extremo más meridional de su distribución en este período, que probablemente alcanzó coincidiendo con una pulsación climática fría. En el caso de B. priscus, de acuerdo con Sesé \& Soto (2002a) esta especie está bien adaptada, aunque no exclusivamente, a condiciones de estepa fría.

Por consiguiente, la presencia en $\mathrm{H}-02$ de $M$. arvalis junto con $B$. priscus, sugieren unas condiciones climáticas menos cálidas que las que existieron durante la formación de otros yacimientos de las terrazas del Jarama y Manzanares en los que estas especies están ausentes. Teniendo en cuenta la acotación a la cronología del yacimiento aportada por la presencia y estadio evolutivo de M. brecciensis, la formación de H-02 debió producirse en el contexto de un estadio isotópico par, MIS 8 o, con más probabilidad, MIS 6 si consideramos que Valdocarros se sitúa en la transición MIS 8/MIS 7 (Panera et al., 2011; Blain et al., 2012).

\section{Análisis de la concordancia entre la interpretación biocronológica y las dataciones numéricas de los yacimientos del ETB}

Los trabajos previos de Arcos Fernández et al. (2008) y de Domínguez Alonso et al. (2009; Figs. 2 y 3 ) ofrecieron cronologías de los yacimientos del ETB, obtenidas por TL por Quaternary TL Surveys, hoy desaparecida, en las secuencias estratigráficas 1, 2 y 3, de arriba a abajo (Fig. 2). La secuencia 3, que contiene el yacimiento $\mathrm{H}-03$ a su techo, tendría una edad más antigua que el MIS 5 $(>125 \mathrm{ka})$. La secuencia 2 tendría hacia su muro, en la posición estratigráfica de $\mathrm{H}-02$, una edad de $80 \mathrm{ka}$ (media de 84,6 y 74,9 ka) situando este yacimiento en el MIS 5a. A techo de la secuencia 2 se ha obtenido una fecha de $56,8 \pm 4 \mathrm{ka}$, principios del MIS 3 (60-24 ka ) o finales del MIS 4. El techo de la secuencia 1 es más reciente, de edad holocena en su parte superior con yacimientos arqueológicos desde épocas neolíticas a altomedievales (Domínguez Alonso et al., 2009).
En el caso del yacimiento H-03, la asociación de mamíferos identificada es poco significativa desde el punto de vista de biocronológico y define para el yacimiento un intervalo cronológico muy amplio, que comprende la segunda mitad del Pleistoceno Medio y todo el Pleistoceno Superior. La fecha obtenida en este yacimiento, de $>125 \mathrm{ka}$, es congruente con la aproximación a su edad a partir de la interpretación biocronológica y la acota dentro del Pleistoceno Medio.

La interpretación biocronológica de la asociación de mamíferos de H-02, basada en la presencia de $M$. arvalis $\mathrm{y}$, sobre todo, en la identificación y estadio evolutivo de $M$. brecciensis, sitúa este yacimiento en el Pleistoceno Medio, en un momento anterior al inicio del MIS 5 y más joven que el MIS 9, y contraviene por una parte la edad establecida por radiometría TL para la base de la secuencia 2 y, por otra, pone en duda la edad del techo de la secuencia 2 de $56,8 \pm 4 \mathrm{ka}$, a no ser que la parte superior de esta secuencia de unos $2,5 \mathrm{~m}$ de espesor, constituida por arenas y fangos de llanura de inundación, fuera una nueva unidad estratigráfica en disconformidad sobre los términos fluviales inferiores que contienen el yacimiento $\mathrm{H}-02$.

Las situaciones de discrepancia entre los contextos cronológicos obtenidos a partir de la interpretación de las asociaciones de mamíferos y las dataciones numéricas resultado de la aplicación de diferentes métodos de datación son frecuentes. En estos casos, y errores aparte, se plantean dos alternativas: la reconsideración del marco biocronológico que ha servido de base para la interpretación de la edad del yacimiento, o la reevaluación del significado de las dataciones numéricas obtenidas.

En relación al yacimiento $\mathrm{H}-02$, el marco de referencia utilizado para precisar su edad ha sido principalmente la evolución del subgénero Iberomys en la península ibérica. A partir de los trabajos de distintos autores (Sesé \& Sevilla, 1996; Cuenca-Bescós et al., 2010b; Laplana \& Sevilla, 2013, entre otros), existe coincidencia tanto a la hora de establecer un vínculo antecesor-descendiente entre $M$. brecciensis y M. cabrerae, como en relación con la distribución temporal de cada especie. M. brecciensis es una especie característica del Pleistoceno Medio, mientras que M. cabrerae lo es del Pleistoceno Superior y Holoceno y sobrevive en la actualidad. La sustitución 
de una especie por otra se produciría en la península ibérica en el MIS 6, si se aceptan como válidas las dataciones de la última población conocida de M. brecciensis, la de la Sala de los Huesos de la cueva de Maltravieso (el nivel con restos de micromamíferos se encuentra entre dos planchas estalagmíticas datadas respectivamente en $117+17 /-14 \mathrm{ka}$ y 183+14/-12 ka según Hanquet, 2011) y la primera de $M$. cabrerae (que, de acuerdo con las interpretaciones asumidas en este trabajo, sería la Arriaga I, datado en $>134$ ka según Silva et al., 2013 o, en su defecto, las de los yacimientos de la Cueva del Camino y PRERESA, datados en $c .90 \mathrm{ka}$ en Arsuaga et al., 2012, у c. 85 ka BP en Sesé et al., 2011 b, respectivamente). La presencia de $M$. brecciensis en H-02 implica, con este referente, una edad Pleistoceno Medio para el yacimiento (excluyendo la base de esta época, momento en el que está presente su antecesora $M$. huescarensis según Laplana \& Cuenca-Bescós, 1997) y pone en cuestión las fechas de $84,6+12,6 /-11,2$ ka y de $74,9+10,2 /-9,2$ ka obtenidas en el yacimiento $\mathrm{H}-02$.

De acuerdo con este marco de referencia, estas dataciones deben ser tenidas en cuenta por tanto como una estimación mínima de la edad del yacimiento, siendo su edad real más antigua, como se ha razonado anteriormente. No es posible en la actualidad establecer el motivo de la obtención de fechas excesivamente recientes con la aplicación del método de la termoluminiscencia para la datación de este yacimiento.

Otros datos conocidos confirman la existencia de secuencias estratigráficas de edad Pleistoceno Medio en la base de los depósitos aflorantes de la TCB. Es el caso de Los Estragales (Fig. 1), localizado unos 3 $\mathrm{km}$ aguas abajo del ETB, y con edades por TL $>125$ ka BP (Pérez-González et al., 2008), situándose el muro de ellas en cotas topográficas por debajo del cauce y llanura aluvial actual. En las canteras de arenas de Arriaga (Fig. 1) se han datado por TL facies fluviales también aflorantes con edades más antiguas de $134 \mathrm{ka}$ BP (Silva et al., 2013). En esta posición geográfica del río Manzanares, próxima a la desembocadura en el río Jarama, hay que hacer notar que la TCB tiene espesores en cota por debajo de la llanura aluvial de decenas de metros (Uribelarrea, 2008). Los yacimientos de los areneros de Arriaga igualmente contienen Palaeoloxodon antiquus y una asociación de micromamíferos similar a la del yacimiento $\mathrm{H}-02$ de la ETB, salvo por la presencia, en nuestra opinión, de M. cabrerae en Arriaga I en lugar de $M$. brecciensis, presente en $\mathrm{H}-02$. El registro de estas especies en cada uno de los yacimientos implica una edad más reciente para Arriaga I que para $\mathrm{H}-02$.

\section{Conclusiones}

Las evidencias aportadas en este trabajo, basadas en la interpretación biocronológica del conjunto de especies de mamíferos identificado en el yacimiento $\mathrm{H}-02$, y en particular, del significado cronológico de la especie $M$. brecciensis presente en el mismo, cuestionan razonablemente la edad de finales del primer tercio del Pleistoceno Superior propuesta en Domínguez Alonso et al. (2009) para el yacimiento H-02 y para la base de la secuencia 2 del ETB. Los datos biocronológicos indican una edad para $\mathrm{H}-02$ dentro del último cuarto del Pleistoceno Medio, más moderna que Áridos 1 y que Valdocarros y más antigua que Arriaga I. Las cronologías numéricas obtenidas por TL de 74,9 y 84,6 ka serían por consiguiente edades mínimas del yacimiento. Sin embargo, subsiste el problema de la asignación cronológica de las facies de arenas y arcillas del techo de la secuencia 2 (Fig. 2) datada asimismo por TL en $56,8 \pm 4$ ka. Una explicación posible es que el techo de la secuencia 2 esté en disconformidad sobre los términos medioinferiores de la misma, o que el dato numérico de 56,8 ka (MIS3-4), represente una edad mínima al igual que la propuesta en este trabajo para el yacimiento H-02. En este último caso, la secuencia 2 podría ser toda ella del Pleistoceno Medio. La secuencia 1, a techo sería del Pleistoceno Superior-Holoceno.

Finalmente, conviene indicar que esta sección del ETB con las secuencias estratigráficas 1, 2 y 3 es de la mayor importancia para situar cronológicamente las terrazas escalonadas de aguas arriba y los depósitos superpuestos y hundidos de la TCB, aguas abajo hasta la desembocadura en el río Jarama.

\section{AGRADECIMIENTOS}

Agradecemos a Carmen Cacho y a Juan Antonio Martos, del Museo Arqueológico Nacional, el haber facilitado el acceso al primero de los autores a la muestra de Microtus brecciensis de Áridos 1, depositada en dicha institución. Queremos expresar 
nuestro agradecimiento a los directores de la intervención arqueológica, Nuria Gallego Lletjós e Iván González García, así como a la Dirección General de Patrimonio Histórico de la Comunidad de Madrid, cuyos técnicos supervisaron dicha intervención. Los comentarios de los revisores C. Sesé y P. G. Silva han contribuido a mejorar el manuscrito original. Este trabajo es una aportación al proyecto I+D S2010/BMD-2330 de la Consejería de Educación de la Comunidad de Madrid.

\section{Referencias}

Álvarez Catalán, V.; Arcos Fernández, S.; Gallego Lletjos, N.; Gil Ortiz, C.; González García, I.; Herráez Igualador, E.; Ruiz Zapata, B. \& Yravedra Sanz de los Terreros, J. (2009). Yacimiento Paleolítico del Estanque de Tormentas de Butarque. 718-05-H-02. In: Actas de las Cuartas Jornadas de Patrimonio Arqueológico en la Comunidad de Madrid (Benet Jordana, N. \& Benito, J.E., Coords.), 333-338.

Álvarez-Lao, D.J. (2014). The Jou Puerta cave (Asturias, NW Spain): a MIS3 large mammal assemblage with mixture of cold and temperate elements. Palaeogeography, Palaeoclimatology, Palaeoecology, 393: 1-19. http://dx.doi.org/10.1016/j.palaeo.2013.10.011.

Álvarez-Lao, D. \& García, N. (2010). Chronological distribution of Pleistocene cold-adapted large mammal faunas in the Iberian Peninsula. Quaternary International, 212: 120-128. http://dx.doi.org/10.1016/j. quaint.2009.02.029.

Álvarez-Lao, D. \& García, N. (2012). Comparative revision of the Iberian woolly mammoth (Mammuthus primigenius) record into a European context. Quaternary Science Reviews, 32: 64-74. http://dx.doi. org/10.1016/j.quascirev.2011.11.004.

Arcos Fernández, S.; Gallego Lletjós, N.; Gil Ortiz, C.; González García, I. \& Sainz de los Terreros, Y. (2008). Geoarqueología del yacimiento paleolítico del Estanque de Tormentas de Butarque (Villaverde, Madrid). I Jornadas de Jóvenes en Investigación Arqueológica, I: 135-140.

Arcos Fernández, S.; Gallego, N.; Gil, C.; González, I. \& Yravedra, J. (2011). El nivel 4 (arcillas) del yacimiento paleolítico del Estanque de Tormentas de Butarque (Villaverde, Madrid). In: Actas de las Quintas Jornadas de Patrimonio Arqueológico en la Comunidad de Madrid (Santonja, M., Coord.): 323-327.

Arsuaga, J.L.; Baquedano, E.; Pérez-González, A.; Sala, N.; Quam, R.M.; Rodríguez, L.; García, R.; García, N.; Álvarez-Lao, D.; Laplana, C.; Huguet, R.; Sevilla, P.; Maldonado, E.; Blain, H.-A.; RuizZapata, M.B.; Sala, P.; Gil-García, M.J.; Uzquiano, P.; Pantoja, A. \& Márquez, B. (2012). Understanding the ancient habitats of the last-interglacial (late MIS 5) Neanderthals of central Iberia: Palaeoenvironmental and taphonomic evidence from the
Cueva del Camino (Spain) site. Quaternary International, 275: 55-75. http://dx.doi.org/10.1016/j. quaint.2012.04.019.

Ayarzagüeña, J. \& López Martínez, N. (1976). Estudio filogenético y comparativo de Microtus cabrerae y Microtus brecciensis. Doñana, Acta Vertebrata, 3 (2): 181-204.

Barroso Ruiz, C.; Desclaux, E. \& Abbassi, M. (2006). Les rongeurs (Mammalia, Rodentia) du Pléistocène supérieur de la Grotte du Boquete de Zafarraya. In: La Grotte du Boquete de Zafarraya. Consejería de Cultura, Junta de Andalucía (Barroso Ruiz, C. \& de Lumley, H., Dir.): 927-977.

Blain, H.-A.; Panera, J.; Uribelarrea, D.; Rubio-Jara, S. \& Pérez-González, A. (2012). Characterization of a rapid climate shift at the MIS 8/7 transition in central Spain (Valdocarros II, Autonomous Region of Madrid) by means of the herpetological assemblages. Quaternary Science Reviews, 47: 73-81. http:// dx.doi.org/10.1016/j.quascirev.2012.05.021.

Cabrera-Millet, M.; López-Martínez, N. \& Michaux, J. (1982). Un exemple de lignée endémique iberoccitane, les campagnols Microtus brecciensis et Microtus cabrerae (Mammalia, Rodentia): étude phylogénétique et contexte écologique d'un phénomène évolutif récent. In: Actes du Symposium paléontologique G. Cuvier (Buffetaut, E., Mazin, J.M. \& Salomon, E., Eds.), Montbéliard: 525-538.

Cuenca-Bescós, G.; Canudo, J.I. \& Laplana, C. (1995). Los arvicólidos (Rodentia, Mammalia) de los niveles inferiores de Gran Dolina (Pleistoceno Inferior, Atapuerca, Burgos, España). Revista de la Sociedad Española de Paleontología, 10 (2): 202-218.

Cuenca Bescós, G. \& Laplana, C. (1995). Evolución de Iberomys (Arvicolidae, Rodentia, Mammalia) durante el Cuaternario español. Resúmenes XI Jornadas de Paleontología, 69-72.

Cuenca-Bescós, G.; Martínez, I.; Mazo, C.; Sauqué, V.; Ramón del Río, D.; Rabal Garcés, R. \& Canudo, J.I. (2010a). Nuevo yacimiento de vertebrados del Cuaternario del sur del Ebro en Aguilón, Zaragoza, España. III Congreso Ibérico de Paleontología, Lisboa: 6-8.

Cuenca-Bescós, G.; Rofes, J.; López-García, J.M.; Blain, H.-A.; De Marfá, R.J.; Galindo-Pellicena, M.A.; Bennásar-Serra, M.L.; Melero-Rubio, M.; Arsuaga, J.L.; Bermúdez de Castro, J.M. \& Carbonell, E. (2010b). Biochronology of Spanish Quaternary small vertebrate faunas. Quaternary International, 212: 109-119. http://dx.doi.org/10.1016/j.quaint.2009. 06.007 .

Cuenca-Bescós, G.; López-García, J.M.; Galindo-Pellicena, M.A.; García-Perea, R.; Gisbert, J.; Rofes, J. \& Ventura, J. (2014). Pleistocene history of Iberomys, an endangered endemic rodent from southwestern Europe. Integrative Zoology, 9: 481-497. http://dx.doi. org/10.1111/1749-4877.12053. 
Espinoza, E. \& Mann, M.J. (1993). The history and significance of the Schreger Pattern in Proboscidean ivory characterization. Journal of the American Institute for Conservation, 32: 241-248. http://dx.doi. org/10.1179/019713693806124866.

Engels, H. (1972). Kleinsauger aus Portugal. Bonner Zoologische Beiträge, 23: 79-86.

Fuentes Jiménez, J.M. (1989). Roedores del yacimiento solutrense "Cueva de los Ojos" (Cozvíjar, Granada). Resúmenes de las V Jornadas de Paleontología, Valencia, s.p.

Gamazo, M. (1982). Prospecciones en las terrazas de la margen derecha del río Manzanares (Getafe y RivasVaciamadrid). Noticiario Arqueológico Hispánico, 14: 7-148.

García, C. (1979). Los roedores de Cueva Horá (Darro, Granada). Nuevos datos sobre la fauna del Pleistoceno Superior de Andalucía. Antropología y Paleoecología Humana, 1: 79-83.

Gil, E. (1985). Estudio de los micromamíferos (Rodentia) de la Cueva del Coscojar, Mora de Rubielos, Teruel. Teruel, 73: 37-58.

Gil, E. (1988). Los micromamíferos (Insectivora, Rodentia). In: Estudio geológico y paleontológico de la Cueva de las Graderas (Molinos, Teruel) (Liñán, E., Coord.). Memorias del Museo Paleontológico de la Universidad de Zaragoza, 3 (1): 31-42.

Gil, E. (1997). Bioestratigrafía y evolución de Microtus brecciensis en los yacimientos del Pleistoceno de Atapuerca (Burgos, España). Geogaceta, 21: 113-116.

González-Esteban, J. \& Villate, I. (2007). Microtus arvalis (Pallas, 1778). In: Atlas y Libro Rojo de los Mamíferos Terrestres de España (Palomo, L.J., Gisbert, J. \& Blanco, J.C., Eds.). Dirección General para la Biodiversidad -SECEM-SECEMU, Madrid, 426-428.

González-Ramón, A.; Andreo, B.; Ruiz-Bustos, A.; Richards, D.A.; López-Sáez, J.A. \& Alba-Sánchez, F. (2012). Late Quaternary paleoenvironmental record from a sedimentary fill in Cucú cave, Almería, SE Spain. Quaternary Research, 77: 264-272. http:// dx.doi.org/10.1016/j.yqres.2011.12.001.

Goy, J.L.; Pérez-González, A. \& Zazo, C. (1989). Cartografía y Memoria del Cuaternario y Geomorfología, Hoja de Madrid (559). Mapa Geológico de España. Escala 1:50.000. 2 ${ }^{\mathrm{a}}$ Serie (MAGNA). IGME. Servicio de Publicaciones del Ministerio de Industria. Madrid.

Guillem Calatayud, P. (2009). Los micromamíferos (Rodentia, Insectivora y Chiroptera) de la secuencia holocena de la Cova de les Cendres. In: La Cova de Les Cendres (Bernabeu Aubán, J. \& Molina Balaguer, L., Coords.): 189-193.

Gusi, F.; Olària, C.; Ollé, A.; Saladié, P.; Vallverdú, J.; Cáceres, I.; van der Made, J.; Expósito, I.; Burjachs, F.; López-Polín, L.; Lorenzo, C.; Bennàsar, M.; Salazar-García, D.M. \& Carbonell, E. (2013). La Cova de Dalt del Tossal de la Font (Vilafamés,
Castellón): conclusiones preliminares de las intervenciones arqueológicas (1982-1987/2004-2012). Quaderns de Prehistòria i Arqueologia de Castelló, 31: 17-37.

Hammer, Ø.; Harper, D.A.T. \& Ryan, P.D. (2001). PAST: Paleontological Statistics Software Package for Education and Data Analysis. Palaeontologia Electronica, 4 (1): 9pp.

Hanquet, C. (2011). Évolution des paléoenvironnements et des paléoclimats au Pléistocène moyen, en Europe méridionale, d'après les faunes de micromammifères. Thèse Université Montpellier III-Paul Valéry.

Herráez Igualador, E. \& Álvarez Catalán, V. (2007). Estudio de la microfauna encontrada en las obras de construcción del Estanque de Tormentas de Butarque. Geolineal, informe inédito, $64 \mathrm{pp}$.

Jeannet, M. (2000). Gruta da Figueira Brava. Les rongeurs. Memorias da Academia das Ciencias de Lisboa, Classe de Ciencias, 38: 179-243.

Laplana, C. \& Cuenca-Bescós, G. (1997). Los arvicólidos (Arvicolidae, Rodentia) del límite Pleistoceno Inferior-Medio en el relleno cárstico Trinchera Dolina (Sierra de Atapuerca, Burgos, España). In: XII Jornadas de Paleontología. Libro de resúmenes y comunicaciones: 192-195.

Laplana, C. \& Cuenca-Bescós, G. (1998). Precisiones sobre la sistemática de Iberomys (Arvicolidae, Rodentia, Mammalia) del Pleistoceno Inferior de la Sierra de Atapuerca. Geogaceta, 24: 183-186.

Laplana, C. \& Sevilla, P. (2013). Documenting the biogeographic history of Microtus cabrerae Thomas, 1906 (Arvicolinae, Rodentia, Mammalia) through its fossil record. Mammal Review, 43: 309-332. http:// dx.doi.org/10.1111/mam.12003.

Laplana, C.; Blain, H.-A.; Sevilla, P.; Arsuaga, J.L.; Baquedano, E. \& Pérez-González, A. (2013). Un assemblage de petits vertébrés hautement diversifié de la fin du MIS5 dans un environnement montagnard au centre de l'Espagne (Cueva del Camino, Pinilla del Valle, Communauté Autonome de Madrid). Quaternaire, 24 (2): 207-216. http:// dx.doi.org/10.4000/quaternaire.6617.

López García, J.M. (2011). Los micromamíferos del Pleistoceno Superior en la Península Ibérica. Evoluión de la diversidad taxonómica y cambios paleoambientales y paleoclimáticos. Editorial Académica Española, 416 pp.

López-Martínez, N. (1980). Los micromamíferos (Rodentia, Insectivora, Lagomorpha y Chiroptera) del sitio de ocupación achelense de Aridos-1 (Arganda, Madrid). In: Ocupaciones achelenses en el valle del Jarama (Santonja, M.; López Martínez, N. \& Pérez-González, A., Eds.). Publicaciones de la Excelentísima Diputación Provincial de Madrid: 161-202.

Luque-Larena, J.J.; Mougeot, F.; Viñuela, J.; Jareño, D.; Arroyo, L.; Lambin, X. \& Arroyo, B. (2013). Recent large-scale range expansion and outbreaks of the 
common vole (Microtus arvalis) in NW Spain. Basic and Applied Ecology, 14: 432-441. http://dx.doi. org/10.1016/j.baae.2013.04.006.

Medina-Lara, F.; Barroso-Ruiz, C.; Sanchidrian-Torti, J.L. \& Ruiz-Bustos, A. (1986). Avance al estudio de los niveles musterienses de la cueva de Zafarraya, Alcaucín, Málaga (excavaciones de 1981-83). Homenaje a Luis Siret (1983-1984). Junta de Andalucía: 94-105.

Olària, C.; Gusi, F.; Carbonell, E.; Ollé, A.; Vallverdú, J.; Allué, E.; Bennàsar, L.; Bischoff, J.L.; Burjachs, F.; Cáceres, I.; Expósito, I.; López, L.; Saldié, P. \& Vergés, J.M. (2005). Noves intervencions al jaciment plistocènic de la Cova de Dalt del Tossal de la Font (Vilafamés, Castelló). Quaderns de Prehistoria i Arqueologia de Castelló, 24: 9-26.

Panera, J.; Pérez-González, A.; Rubio, S. \& Sesé, C. (2005). El yacimiento paleolítico de HAT en el valle del Jarama: una aportación de Cuaternario de la cuenca de Madrid al debate sobre el inicio del Paleolítico medio. In: Geoarqueología y Patrimonio en la Península Ibérica y el entorno mediterráneo (Santonja, M, Pérez-González, A. \& Machado, M.J., Eds.) ADEMA. Soria: 251-260.

Panera, J.; Torres, T.; Pérez-González, A.; Ortiz, J.E.; Rubio-Jara, S. \& Uribelarrea del Val, D. (2011). Geocronología de la Terraza Compleja de Arganda en el valle de río Jarama (Madrid, España). Estudios geológicos, 67 (2): 495-504. http://dx.doi.org/10.3989/ egeol.40550.204.

Paunesco, A.C. \& Brunet-Lecomte, P. (2005). Analyse odontométrique du sous-genre Microtus (Iberomys) Chaline, 1972 (Rodentia, Arvicolinae) du sud de la France au Pléistocène moyen et supérieur. Bulletin du Musée d'Anthropologie Prehistorique de Monaco, 45: 31-39.

Pérez-González, A. (1971). Estudio de los procesos de hundimiento en el valle del río Jarama y sus terrazas (nota preliminar). Estudios Geológicos, 27 (4): 317-324.

Pérez-González, A.; Rubio Jara, S.; Uribelarrea, D. \& Panera, J. (2008). Geocronología de la sucesión arqueoestratigráfica de Los Estragales en la Terraza Compleja de Butarque (Valle del río Manzanares, Madrid). Geogaceta, 45: 39-42.

Portero, J. \& Pérez-González, A. (1990). Hoja y Memoria del Mapa Geológico de España, E. 1:50.000. Alcalá de Henares (560), ITGE.

Povoas, L.; Zilhao, J.; Chaline, J. \& Brunet-Lecomte, P. (1992). La faune de rongeurs du Pleistocène superieur de la Grotte de Caldeirao (Tomar, Portugal). Quaternaire, 3 (1): 40-47. http://dx.doi.org/10.3406/ quate.1992.1971.

Rey, J.M. (1973). Notas sobre mastozoología Ibérica, I. Las características biométricas y morfológicas del topillo campesino, Microtus Arvalis Asturianus del Sistema Ibérico. Boletín de la Real Sociedad Española de Historia Natural (Biol.), 71: 283-297.
Ros-Montoya, S. (2010). Los proboscídeos del PlioPleistoceno de la Cuenca de Guadix Baza y Granada. Tesis Doctoral, Universidad de Granada, 403 pp.

Rubio Jara, S.; Panera Gallego, J. \& Martos Romero, J.A. (2002). La modernización del Paleolítico en los valles del Manzanares y Jarama: 1970-1985. In: Bifaces y elefantes. La investigación del Paleolítico Inferior en Madrid (Panera, J. \& Rubio Jara, S., Eds.). Zona Arqueológica, 1: 215-241.

Ruiz Bustos, A. (2000). Estudio paleoecológico de los sedimentos con presencia del hombre de Neandertal en la Cueva de la Carihuela (Píñar, Granada). Ayuntamiento de Pínar (Granada), 91 pp.

Ruiz Bustos, A. \& García Sánchez, M. (1977). Las condiciones ecológicas del Musteriense en las depresiones granadinas. La fauna de micromamíferos en la cueva de La Carigüela (Piñar, Granada). Cuadernos de Prehistoria de la Universidad de Granada, 2: 7-17.

Ruiz Bustos, A. \& Riquelme Cantal, J.A. (1999). Análisis sistemático y ecológico de los roedores del Pleistoceno Superior procedentes de la Cueva de las Ventanas (Granada, España): In: Contribución del estudio científico de las cavidades cársticas al conocimiento geológico (Andreo, B., Carrasco, F. \& Durán, J.J., Eds.). Patronato de la Cueva de Nerja (Málaga): 277-288.

Ruiz Bustos, A.; Toro Moyano, I.; Martín Suárez, E. \& Almohalla Gallego, M. (1982). Procesos evolutivos durante el Cuaternario medio y superior en las poblaciones de pequeños mamíferos del sur de la Península Ibérica. Condiciones climáticas que implican e importancia bioestratigráfica. Cuadernos de Prehistoria de la Universidad de Granada, 7: 9-35.

Rus, I., \& Vega, G. (1984). El yacimiento de Arriaga II: Problemas de una definición actual de los suelos de ocupación. Primeras Jornadas de Metodología e Investigación Prehistórica (Soria, 1981): 387-404.

Santonja, M.; López Martínez, N. \& Pérez-González, A. (Eds.) (1980). Ocupaciones Achelenses en el Valle del Jarama (Arganda, Madrid). Arqueología y Paleoecología, 1. Diputación Provincial de Madrid, 352 pp.

Sesé, C. \& López-Martínez, N. (2013). Nuevos datos paleontológicos del Pleistoceno en el Valle del Manzanares (Madrid, España): Los micromamíferos del yacimiento del Arenero de Arriaga. Estudios Geológicos, 69 (2): 271-282. http://dx.doi.org/10.3989/ egeol.41318.270.

Sesé, C. \& Sevilla, P. (1996). Los micromamíferos del Cuaternario peninsular español: cronoestratigrafía e implicaciones bioestratigráficas. Revista Española de Paleontología, ${ }^{\circ}$ extraordinario: 278-287.

Sesé, C.; Panera, J.; Rubio-Jara, S. \& Pérez-González, A. (2011a). Micromamíferos del Pleistoceno Medio y Pleistoceno Superior en el Valle del Jarama: yacimientos de Valdocarros y HAT (Madrid, España). Estudios Geológicos, 67 (1): 131-151. http://dx.doi. org/10.3989/egeol.40290.127. 
Sesé, C.; Rubio-Jara, S.; Panera, J. \& Pérez-González, A. (2011b). Micromamíferos del Pleistoceno Superior del yacimiento de PRERESA en el valle del Manzanares y su contribución a la reconstrucción paleoambiental de la cuenca de Madrid durante el Pleistoceno. Estudios Geológicos, 67 (2): 471-494. http://dx.doi.org/10.3989/egeol.40516.203.

Silva, P.G.; López Recio, M.; Cuartero, F.; Baena, J.; Tapias, F.; Manzano, I.; Martín, D.; Morín, J. \& Roquero, E. (2012). Contexto geomorfológico y principales rasgos tecnológicos de nuevos yacimientos del Pleistoceno Medio y Superior en el Valle Inferior del Manzanares (Madrid, España). Estudios Geológicos, 68 (1): 58-89. http://dx.doi.org/10.3989/egeol.40338.134.

Silva, P.G.; López-Recio, M.; Tapias, F.; Roquero, E.; Morín, J.; Rus, I.; Carrasco-García, P.; Giner-Robles, J.L.; Rodríguez-Pascua, M.A. \& Pérez-López, R. (2013). Stratigraphy of the Arriaga Palaeolithic sites. Implications for the geomorphological evolution recorded by thickened fluvial sequences within the Manzanares River valley (Madrid Neogene Basin, Central Spain). Geomorphology, 196: 138-161. http://dx.doi.org/10.1016/j.geomorph.2012.10.019.

Toni, I. \& Molero, G. (1990). Los roedores (Rodentia, Mammalia) del yacimiento cuaternario de Pinilla del Valle (Madrid). Actas de las IV Jornadas de Paleontología, Salamanca, 1988: 359-373.
Tormo Cuñat, C. (2010). Los roedores e insectívoros de los niveles gravetienses de la Cova de les Cendres (Teulada-Moraria, Alicante). Implicaciones paleoclimáticas. Archivo de Prehistoria Levantina, 28: 47-71.

Uribelarrea, D. (2008). Dinámica y evolución de las llanuras aluviales de los ríos Manzanares, Jarama y Tajo, entre las ciudades de Madrid y Toledo. Tesis Doctoral. Departamento de Geodinámica, Facultad de Ciencias Geológicas, Universidad Complutense de Madrid, 344 pp.

Van der Meulen, A. (1973). Middle Pleistocene Smaller Mammals from the Monte Peglia (Orvieto, Italy), with Special Reference to the Phylogeny of Microtus (Arvicolidae, Rodentia). Quaternaria, 17: $1-144$.

Yravedra, J. (2006). Análisis Zooarqueológico y Tafonómico de los niveles pleistocenos del yacimiento Paleolítico H03 (Villaverde Bajo, Madrid). Inédito. AREA, Madrid, 30 pp.

Yravedra, J.; Rubio-Jara, S.; Panera, J.; Uribelarrea, D. \& Pérez-González, A. (2012). Elephants and subsistence. Evidence of the human exploitation of extremely large mammal bones from the Middle Palaeolithic site of Preresa (Madrid, Spain). Journal of Archaeological Science 39: 1063-1071. http://dx. doi.org/10.1016/j.jas.2011.12.004. 